\title{
Portuguese and French Validation of Need for Drama (NFD) Scale in Consumer Behavior: An Abstract
}

\author{
Bruno Morgado Ferreira and Cindy Caldara
}

\begin{abstract}
This article aims at testing and validating the use of a scale recently developed in psychology: the Need for Drama (NFD, Frankowski et al. 2016). The transposition of the scale led on Portuguese and French consumers, extracting the scale from its original context (workplace and interpersonal relationships with US respondents). The results concur to assert the good validity of the scale and of its structure on both populations. Interestingly though, both samples lead to the conclusion that in these countries, the results are correlated with gender, when it was not the case in the American original study. The research avenues and managerial implications are discussed.
\end{abstract}

Acknowledgment This research was partially funded by the AMS-AFM Research Grant.

B. M. Ferreira $(\bowtie)$

Polytechnic Institute of Viseu, Viseu, Portugal

e-mail: morgado.ferreira@estgv.ipv.pt

C. Caldara

University of Grenoble Alpes-CERAG, Grenoble, France

e-mail: cindy.caldara@iut2.univ-grenoble-alpes.fr 